7 pages, 3 figures

\title{
Tunneling transport through multi-electrons states in coupled quantum dots with Coulomb correlations
}

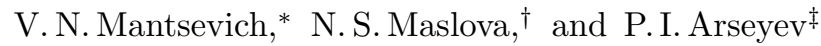 \\ Moscow State University, Department of Physics, \\ 119991 Moscow, Russia \\ P.N. Lebedev Physical institute of RAS, \\ 119991, Moscow, Russia
}

(Dated: June 2, 2021)

\begin{abstract}
We investigated the peculiarities of non-equilibrium charge configurations in the system of two strongly coupled quantum dots (QDs) weakly connected to the reservoirs in the presence of Coulomb correlations. We revealed that total electron occupation demonstrates in some cases significant decreasing with increasing of applied bias - contrary to the situation when Coulomb correlations are absent and found well pronounced ranges of system parameters where negative tunneling conductivity appears due to the Coulomb correlations.
\end{abstract}

PACS numbers: 73.63.Kv, 73.40.Gk, 73.21.La

Keywords: D. Coulomb correlations; D. Quantum dots; D. Tunneling

\section{INTRODUCTION}

Electron tunneling through the system of coupled QDs in the presence of strong Coulomb correlations is one of the most interesting problems in the solid state physics. The present day experimental technique gives possibility to produce single QDs with a given set of parameters and to create coupled QDs with different spatial geometry [1], [2], [3] . Thereby the main effort in the physics of QDs is devoted to the investigation of non-equilibrium charge states and different charge configurations due to the electrons tunneling [4], [5], [6], 7], [8] through the system of coupled QDs in the presence of strong Coulomb interaction.

One of the most intensively studied problems in this field is tunneling through the single QD [9], [10] and interacting QDs [4], [5], 11], 12] in the Kondo regime, which reveals rich physics for small bias voltage compared to the tunneling rates. It was demonstrated [11] that Coulomb correlations in QDs lead to bistable behavior in the Kondo regime at zero bias voltage. Charge redistribution between different spin configurations in the system of two interacting QDs in the Kondo regime was regarded in [4]. The authors considered the situation when the detuning between the energy levels in the QDs exceeds the dots coupling and on-site Coulomb repulsion is present only in a single dot.

A great attention is also payed to the double QDs as attractive systems for spin-dependent transport [13], 14], [15]. In [13] authors studied transport through double QD both in sequential tunneling and co-tunneling

\footnotetext{
*vmantsev@spmlab.phys.msu.ru

${ }^{\dagger}$ Electronic address: spm@spmlab.phys.msu.ru

$\ddagger_{\text {ars@lpi.ru }}$
}

regimes by means of master equation for density matrix in the basis of exact eigenfunctions and eigenvalues. Unfortunately only transitions between the empty states and states with one and two electrons were considered. Results obtained in [14] and [15] deal with the investigation of transport in the a double QD system weakly coupled to spin-polarized leads. The method of charge and spin transport analysis presented in [14] is based on the Liouville equation for the reduced density matrix in lowest order in the tunneling transitions. Authors analyzed tunneling conductivity and $I-V$ characteristics as a functions of magnetic leads polarization and gate voltage. The system where only one or two electrons can be localized simultaneously due to the specific features of the ferromagnetic leads was investigated in 15$]$.

In the present paper we consider electron tunneling through the coupled QDs in the regime when applied bias can be tuned in a wide range and the on-site Coulomb repulsion can be comparable to the other system parameters. We analyze different charge configurations in the system of two strongly coupled quantum dots (QDs) weakly connected to the reservoirs in the presence of Coulomb correlations in a wide range of applied bias in terms of pseudo operators with constraint [16], [17], [18], [8]. For large values of applied bias Kondo effect is not essential so we neglect any correlations between electron states in the QDs and in the leads. This approximation allows to describe correctly non equilibrium occupation of any single- and multi-electron state due to the tunneling processes. We revealed the presence of negative tunneling conductivity in certain ranges of the applied bias voltage and revealed that total electron occupation demonstrates in some cases significant decreasing with increasing of applied bias - contrary to the situation when Coulomb correlations are absent. 


\section{MODEL}

We consider a system of coupled QDs with the single particle levels $\widetilde{\varepsilon}_{1} \quad \widetilde{\varepsilon}_{2}$ connected to the two leads. The Hamiltonian can be written as:

$$
\begin{aligned}
\hat{H} & =\sum_{\sigma} c_{1 \sigma}^{+} c_{1 \sigma} \widetilde{\varepsilon}_{1}+\sum_{\sigma} c_{2 \sigma}^{+} c_{2 \sigma} \widetilde{\varepsilon}_{2}+U_{1} \widehat{n}_{1 \sigma} \widehat{n}_{1-\sigma}+ \\
& +U_{2} \widehat{n}_{2 \sigma} \widehat{n}_{2-\sigma}+\sum_{\sigma} T\left(c_{1 \sigma}^{+} c_{2 \sigma}+c_{2 \sigma}^{+} c_{1 \sigma}\right)
\end{aligned}
$$

where operator $c_{l \sigma}$ creates an electron in the dot $i$ with spin $\sigma, \widetilde{\varepsilon}_{l}$ is the energy of the single electron level in the dot $i$ and $T$ is the inter-dot tunneling coupling, $n_{l \sigma}=c_{l \sigma}^{+} c_{l \sigma}$ and $U_{1(2)}$ is the on-site Coulomb repulsion of localized electrons. When the coupling between QDs exceeds the value of interaction with the leads, one has to use the basis of exact eigenfunctions and eigenvalues of coupled QDs without the interaction with the leads. In this case all energies of single- and multi-electron states are well known:

One electron in the system: two single electron states with the wave function

$$
\psi_{i}^{\sigma}=\mu_{i} \cdot|0 \uparrow\rangle|00\rangle+\nu_{i} \cdot|00\rangle|0 \uparrow\rangle
$$

Single electron energies and coefficients $\mu_{i}$ and $\nu_{i}$ can be found as an eigenvalues and eigenvectors of matrix:

$$
\left(\begin{array}{cc}
\varepsilon_{1} & -T \\
-T & \varepsilon_{2}
\end{array}\right)
$$

Two electrons in the system: two states with the same spin $\sigma \sigma$ and $-\sigma-\sigma$ and four two-electron states with the opposite spins $\sigma-\sigma$ with the wave function:

$$
\begin{aligned}
\psi_{j}^{\sigma-\sigma} & =\alpha_{j} \cdot|\uparrow \downarrow\rangle|00\rangle+\beta_{k} \cdot|\downarrow 0\rangle|0 \uparrow\rangle+ \\
& +\gamma_{j} \cdot|0 \uparrow\rangle|\downarrow 0\rangle+\delta_{j} \cdot|00\rangle|\uparrow \downarrow\rangle
\end{aligned}
$$

Two electron energies and coefficients $\alpha_{j}, \beta_{j}, \gamma_{j}$ and $\delta_{j}$ are the eigenvalues and eigenvectors of matrix:

$$
\left(\begin{array}{cccc}
2 \varepsilon_{1}+U_{1} & -T & -T & 0 \\
-T & \varepsilon_{1}+\varepsilon_{2} & 0 & -T \\
-T & 0 & \varepsilon_{1}+\varepsilon_{2} & 0 \\
0 & -T & -T & 2 \varepsilon_{2}+U_{2}
\end{array}\right)
$$

Three electrons in the system: two three-electron states with the wave function

$$
\begin{aligned}
\psi_{m}^{\sigma \sigma-\sigma} & =p_{m}|\uparrow \downarrow\rangle|\uparrow\rangle+q_{m}|\uparrow\rangle|\uparrow \downarrow\rangle \\
m & = \pm 1
\end{aligned}
$$

Three electron energies and coefficients $p_{m}$ and $Q_{m}$ can be found as an eigenvalues and eigenvectors of matrix:

$$
\left(\begin{array}{cc}
2 \varepsilon_{1}+\varepsilon_{2}+U_{1} & -T \\
-T & 2 \varepsilon_{2}+\varepsilon_{1}+U_{2}
\end{array}\right)
$$

Four electrons in the system: one four-electron state with energy $E_{I V l}=2 \varepsilon_{1}+2 \varepsilon_{2}+U_{1}+U_{2}$ and wave function

$$
\psi_{l}=|\uparrow \downarrow\rangle|\uparrow \downarrow\rangle
$$

If coupled QDs are connected with the leads of the tunneling contact the number of electrons in the dots changes due to the tunneling processes. Transitions between the states with different number of electrons in the two interacting QDs can be analyzed in terms of pseudoparticle operators with constraint on the physical states (the number of pseudo-particles). Consequently, the electron operator $c_{\sigma l}^{+}(l=1,2)$ can be written in terms of pseudo-particle operators as:

$$
\begin{aligned}
c_{\sigma l}^{+} & =\sum_{i} X_{i}^{\sigma l} f_{\sigma i}^{+} b+\sum_{j, i} Y_{j i}^{\sigma-\sigma l} d_{j}^{+\sigma-\sigma} f_{i-\sigma}+ \\
& +\sum_{j, i} Y_{i}^{\sigma \sigma l} d^{+\sigma \sigma} f_{i \sigma}+\sum_{m, j} Z_{m j}^{\sigma \sigma-\sigma l} \psi_{m-\sigma}^{+} d_{j}^{\sigma-\sigma}+ \\
& +\sum_{m} Z_{m}^{\sigma-\sigma-\sigma l} \psi_{m \sigma}^{+} d^{-\sigma-\sigma}+\sum_{m} W_{m}^{\sigma-\sigma-\sigma l} \varphi^{+} \psi_{m \sigma}
\end{aligned}
$$

where $f_{\sigma}^{+}\left(f_{\sigma}\right)$ and $\psi_{\sigma}^{+}\left(\psi_{\sigma}\right)$ - are pseudo-fermion creation (annihilation) operators for the electronic states with one and three electrons correspondingly. $b^{+}(b)$, $d_{\sigma}^{+}\left(d_{\sigma}\right)$ and $\varphi^{+}(\varphi)$ - are slave boson operators, which correspond to the states without any electrons, with two electrons or four electrons. Operators $\psi_{m-\sigma^{-}}^{+}$describe system configuration with two spin up electrons $\sigma$ and one spin down electron $-\sigma$ in the symmetric and asymmetric states.

Matrix elements $X_{i}^{\sigma l}, Y_{j i}^{\sigma-\sigma l}, Y_{j i}^{\sigma \sigma l}, Z_{m j}^{\sigma \sigma-\sigma l}, Z_{m j}^{\sigma-\sigma-\sigma l}$ and $W_{m}^{\sigma-\sigma-\sigma l}$ can be evaluated as:

$$
\begin{aligned}
X_{i}^{\sigma l} & =\left\langle\psi_{i}^{\sigma}\left|c_{\sigma l}^{+}\right| 0\right\rangle \\
Y_{j i}^{\sigma-\sigma l} & =\left\langle\psi_{j}^{\sigma-\sigma}\left|c_{\sigma i l}^{+}\right| \psi_{i}^{-\sigma}\right\rangle \\
Y_{j i}^{\sigma \sigma l} & =\left\langle\psi_{j}^{\sigma \sigma}\left|c_{\sigma l}^{+}\right| \psi_{i}^{\sigma}\right\rangle \\
Z_{m j}^{\sigma \sigma-\sigma l} & =\left\langle\psi_{m}^{\sigma \sigma-\sigma}\left|c_{\sigma l}^{+}\right| \psi_{j}^{\sigma-\sigma}\right\rangle \\
Z_{m}^{\sigma-\sigma-\sigma l} & =\left\langle\psi_{m}^{\sigma-\sigma-\sigma}\left|c_{\sigma l}^{+}\right| \psi^{-\sigma-\sigma}\right\rangle \\
W_{m}^{\sigma-\sigma-\sigma l} & =\left\langle\psi_{l}^{\sigma \sigma-\sigma-\sigma}\left|c_{\sigma l}^{+}\right| \psi_{m}^{\sigma-\sigma-\sigma}\right\rangle
\end{aligned}
$$

Finally one can easily express matrix elements through the matrixes (3), (5), (7) eigenvectors elements: 


$$
\begin{array}{r}
X_{i}^{\sigma 1}=\mu_{i} ; X_{i}^{\sigma 2}=\nu_{i} \\
Y_{j i}^{\sigma-\sigma 1}=\alpha_{j} \mu_{i}+\beta_{j} \nu_{i} \\
Y_{j i}^{\sigma-\sigma 2}=\delta_{j} \nu_{i}+\gamma_{j} \mu_{i} \\
Y_{j i}^{\sigma \sigma 1}=\nu_{i} ; Y_{j i}^{\sigma \sigma 2}=\mu_{i} \\
Z_{m j}^{\sigma \sigma-\sigma 1}=p_{m} \gamma_{j}+q_{m} \delta_{j} \\
Z_{m j}^{\sigma \sigma-\sigma 2}=p_{m} \alpha_{j}+q_{m} \beta_{j} \\
Z_{m j}^{\sigma-\sigma-\sigma 1}=p_{m} ; Z_{m j}^{\sigma-\sigma-\sigma 1}=q_{m} \\
W_{m}^{\sigma-\sigma-\sigma 1}=q_{m} ; W_{m}^{\sigma-\sigma-\sigma 2}=p_{m}
\end{array}
$$

The constraint on the space of the possible system states have to be taken into account:

$$
\widehat{n}_{b}+\sum_{i \sigma} \widehat{n}_{f i \sigma}+\sum_{j \sigma \sigma^{\prime}} \widehat{n}_{d j}^{\sigma \sigma^{\prime}}+\sum_{m \sigma} \widehat{n}_{\psi m \sigma}+\widehat{n}_{\varphi}=1
$$

Condition (12) means that the appearance of any two pseudo-particles in the system simultaneously is impossible.

Electron filling numbers in the coupled QDs can be expressed in the terms of the pseudo-particles filling numbers:

$$
\begin{aligned}
\widehat{n}_{\sigma}^{e l} & =\sum_{l} c_{\sigma l}^{+} c_{\sigma l}=\sum_{i, l}\left|X_{i}^{\sigma l}\right|^{2} \widehat{n}_{f i \sigma}+\sum_{i, j, l}\left|Y_{j i}^{\sigma-\sigma l}\right|^{2} \widehat{n}_{d j}^{\sigma-\sigma}+ \\
& +\sum_{i, l}\left|Y_{j i}^{\sigma \sigma l}\right|^{2} \widehat{n}_{d j}^{\sigma \sigma}+\sum_{m, j, l}\left|Z_{m j}^{\sigma \sigma-\sigma l}\right|^{2} \widehat{n}_{\psi m-\sigma}+ \\
& +\sum_{m, l}\left|Z_{m j}^{-\sigma-\sigma \sigma l}\right|^{2} \widehat{n}_{\psi m \sigma}+\sum_{m, l}\left|W_{m}^{\sigma-\sigma-\sigma l}\right|^{2} \widehat{n}_{\varphi}
\end{aligned}
$$

Consequently, the Hamiltonian of the system can be written in the terms of the pseudo-particle operators:

$$
\begin{aligned}
\hat{H} & =\hat{H}_{0}+\hat{H}_{t u n} \\
\hat{H}_{0} & =\sum_{i \sigma} \varepsilon_{i} f_{i \sigma}^{+} f_{i \sigma}+\sum_{j \sigma \sigma^{\prime}} E_{I I j}^{\sigma \sigma^{\prime}} d_{j}^{+\sigma \sigma^{\prime}} d_{j}^{\sigma \sigma^{\prime}}+ \\
& +\sum_{m \sigma} E_{I I I}^{m \sigma} \psi_{m \sigma}^{+} \psi_{m \sigma}+E_{I V l} \varphi_{\sigma}^{+} \varphi_{\sigma}+ \\
& +\sum_{k \sigma}\left(\varepsilon_{k \sigma}-e V\right) c_{k \sigma}^{+} c_{k \sigma}+\sum_{p \sigma} \varepsilon_{p \sigma} c_{p \sigma}^{+} c_{p \sigma} \\
\hat{H}_{t u n} & =\sum_{k \sigma} T_{k}\left(c_{k \sigma}^{+} c_{\sigma 1}+c_{\sigma 1}^{+} c_{k \sigma}\right)+(k \leftrightarrow p ; 1 \leftrightarrow 2)
\end{aligned}
$$

where $\varepsilon_{i}, E_{I I j}^{\sigma \sigma^{\prime}}, E_{I I I}^{m \sigma}$ and $E_{I V l \text {-are the energies of the }}$ single-, double-, triple- and quadri-electron states. $\varepsilon_{k(p) \sigma^{-}}$ is the energy of the conduction electrons in the states $k$ and $p$ correspondingly. $c_{k(p) \sigma}^{+} / c_{k(p) \sigma}$ are the creation (annihilation) operators in the leads of the tunneling contact. $T_{k(p)}$-are the tunneling amplitudes, which we assume to be independent on momentum and spin. Indexes $k(p)$ mean only that tunneling takes place from the system of coupled QDs to the conduction electrons in the states $k$ and $p$ correspondingly.

Bilinear combinations of pseudo-particle operators are closely connected with the density matrix elements. So, similar expressions can be obtained from equations for the density matrix evolution but method based on the pseudo particle operators is more compact and convenient. The tunneling current through the proposed system written in terms of the pseudo-particle operators has the form:

$$
\begin{aligned}
\widehat{I}_{k \sigma} & =\sum_{k} \frac{\partial \widehat{n}_{k}}{\partial t}=i\left[\sum_{i k} X_{i}^{\sigma 1} T_{k} c_{k \sigma} f_{i \sigma}^{+} b+\right. \\
& +\sum_{i j k} Y_{j i}^{\sigma-\sigma 1} T_{k} c_{k \sigma} d_{j}^{+\sigma-\sigma} f_{i-\sigma}+\sum_{i j k} Y_{j i}^{\sigma \sigma 1} T_{k} c_{k \sigma} d_{j}^{+\sigma \sigma} f_{i \sigma}+ \\
& +\sum_{m j k} Z_{m j}^{\sigma \sigma-\sigma 1} T_{k} c_{k \sigma} \psi_{m-\sigma}^{+} d_{j}^{\sigma-\sigma}+ \\
& +\sum_{m j k} Z_{m j}^{-\sigma-\sigma \sigma 1} T_{k} c_{k \sigma} \psi_{m \sigma}^{+} d_{j}^{-\sigma-\sigma}+ \\
& \left.+\sum_{m k} W_{m}^{\sigma-\sigma-\sigma 1} T_{k} c_{k \sigma} \varphi^{+} \psi_{m \sigma}-h . c .\right]
\end{aligned}
$$

We set $\hbar=1$ and neglect changes in the electron spectrum and local density of states in the tunneling contact leads, caused by the tunneling current. Therefore equations of motion together with the constraint on the space of the possible system states (pseudo-particles number) (12) give the following equations: 


$$
\begin{array}{r}
I m \sum_{i k} T_{k} X_{i}^{\sigma 1} \cdot\left\langle c_{k \sigma} f_{i \sigma}^{+} b\right\rangle= \\
=\Gamma_{k} \sum_{i}\left[\left(1-n_{k \sigma}\left(\varepsilon_{i}\right)\right) \cdot n_{f i \sigma}-n_{k \sigma}\left(\varepsilon_{i}\right) \cdot n_{b}\right]\left(X_{i}^{\sigma 1}\right)^{2} \\
I m \sum_{i j k} Y_{j i}^{\sigma-\sigma 1} T_{k} \cdot\left\langle c_{k \sigma} d_{j}^{+\sigma-\sigma} f_{i-\sigma}\right\rangle= \\
=\Gamma_{k} \sum_{i j}\left[\left(1-n_{k \sigma}\left(E_{I I j}^{\sigma-\sigma}-\varepsilon_{i-\sigma}\right)\right) \cdot n_{d j}^{\sigma-\sigma}-\right. \\
\left.-n_{k \sigma}\left(E_{I I j}^{\sigma-\sigma}-\varepsilon_{i-\sigma}\right) \cdot n_{f i-\sigma}\right]\left(Y_{j i}^{\sigma-\sigma 1}\right)^{2} \\
I m \sum_{i j k} Y_{j i}^{\sigma \sigma 1} T_{k} \cdot\left\langle c_{k \sigma} d_{j}^{+\sigma \sigma} f_{i \sigma}\right\rangle= \\
=\Gamma_{k} \sum_{i j}\left[\left(1-n_{k \sigma}\left(E_{I I j}^{\sigma \sigma}-\varepsilon_{i \sigma}\right)\right) \cdot n_{d j}^{\sigma \sigma}-\right. \\
\left.-n_{k \sigma}\left(E_{I I j}^{\sigma \sigma}-\varepsilon_{i \sigma}\right) \cdot n_{f i \sigma}\right]\left(Y_{j i}^{\sigma \sigma 1}\right)^{2} \\
I m \sum_{m j k} Z_{m j}^{\sigma \sigma-\sigma 1} T_{k} \cdot\left\langle c_{k \sigma} \psi_{m-\sigma}^{+} d_{j}^{\sigma-\sigma}\right\rangle= \\
=\Gamma_{k} \sum_{m j}\left[\left(1-n_{k \sigma}\left(E_{I I I}^{m-\sigma}-E_{I I j}^{\sigma-\sigma}\right)\right) \cdot n_{\psi m-\sigma}-\right. \\
\left.-n_{k \sigma}\left(E_{I I I}^{m-\sigma}-E_{I I j}^{\sigma-\sigma}\right) \cdot n_{d j}^{\sigma-\sigma}\right]\left(Z_{m j}^{\sigma \sigma-\sigma 1}\right)^{2} \\
I m \sum_{m j k} Z_{m j}^{-\sigma-\sigma \sigma 1} T_{k} \cdot\left\langle c_{k \sigma} \psi_{m \sigma}^{+} d_{j}^{-\sigma-\sigma}\right\rangle= \\
\Gamma_{k} \sum_{m}\left[\left(1-n_{k \sigma}\left(E_{I V l}-E_{I I I}^{m \sigma}\right) \cdot n_{\psi m \sigma}\right]\left(E_{I V l}-E_{I I I}^{m \sigma}\right)\right) \cdot n_{\varphi}- \\
=\Gamma_{k} \sum_{m j}\left[\left(1-n_{k \sigma}\left(E_{I I I}^{m \sigma}-E_{I I j}^{-\sigma-\sigma}\right)\right) \cdot n_{\psi m \sigma}-\right. \\
\left.-n_{k \sigma}\left(E_{I I}^{m \sigma}-E_{I I j}^{-\sigma-\sigma}\right) \cdot n_{d j}^{-\sigma-\sigma}\right]\left(Z_{m j}^{-\sigma-\sigma \sigma 1}\right)^{2} \\
I m \sum_{m}^{\sigma-\sigma-\sigma 1} T_{k} \cdot\left\langle c_{k \sigma} \varphi_{l}^{+} \psi_{m \sigma}\right\rangle=
\end{array}
$$

Tunneling current $I_{k \sigma}$ is determined by the sum of the right hand parts of the equations (16).

Stationary system of equations can be obtained for the pseudo particle filling numbers $n_{f i}, n_{d j}^{\sigma-\sigma}, n_{d}^{\sigma \sigma}, n_{\psi m}$ and $n_{\varphi}:$

$$
\begin{aligned}
& 0=\frac{\partial n_{\varphi}}{\partial t}=-\Gamma_{k} \sum_{m \sigma}\left[-n_{\psi m \sigma} \cdot n_{k \sigma}\left(E_{I V l}-E_{I I I}^{m \sigma}\right)+\right. \\
& \left.+n_{\varphi} \cdot\left(1-n_{k \sigma}\left(E_{I V l}-E_{I I I}^{m \sigma}\right)\right)\right]\left|W_{m}^{\sigma-\sigma-\sigma 1}\right|^{2}+(k, 1 \leftrightarrow p, 2) \\
& 0=\frac{\partial n_{\psi m \sigma}}{\partial t}=-\Gamma_{k} \sum_{j}\left[n_{\psi m \sigma} \cdot\left(1-n_{k-\sigma}\left(E_{I I I}^{m \sigma}-E_{I I j}^{\sigma-\sigma}\right)\right)-\right. \\
& \left.-n_{k-\sigma}\left(E_{I I I}^{m \sigma}-E_{I I j}^{\sigma-\sigma}\right) \cdot n_{d j}^{\sigma-\sigma}\right] \mid\left(\left.Z_{m j}^{\sigma \sigma-\sigma 1}\right|^{2}-\right. \\
& -\Gamma_{k} \sum_{j}\left[\left(1-n_{k \sigma}\left(E_{I I I}^{m \sigma}-E_{I I j}^{-\sigma-\sigma}\right)\right) \cdot n_{\psi m \sigma}-\right. \\
& \left.-n_{d j}^{-\sigma-\sigma} \cdot n_{k \sigma}\left(E_{I I I}^{m \sigma}-E_{I I j}^{-\sigma-\sigma}\right)\right]\left|Z_{m j}^{\sigma-\sigma-\sigma 1}\right|^{2}- \\
& -\Gamma_{k}\left[-\left(1-n_{k \sigma}\left(E_{I V l}-E_{I I I}^{m \sigma}\right)\right) \cdot n_{\varphi}+\right. \\
& \left.+n_{\psi m \sigma} \cdot n_{k \sigma}\left(E_{I V l}-E_{I I I}^{m \sigma}\right)\right]\left|W_{m}^{\sigma-\sigma-\sigma 1}\right|^{2}+(k, 1 \leftrightarrow p, 2) \\
& 0=\frac{\partial n_{d j}^{\sigma \sigma}}{\partial t}=-\Gamma_{k} \sum_{i}\left[\left(1-n_{k \sigma}\left(E_{I I j}^{\sigma \sigma}-\varepsilon_{i}\right)\right) \cdot n_{d j}^{\sigma \sigma}-\right. \\
& \left.-n_{k \sigma}\left(E_{I I j}^{\sigma \sigma}-\varepsilon_{i}\right) \cdot n_{f i \sigma}\right]\left|Y_{j i}^{\sigma \sigma 1}\right|^{2}- \\
& -\Gamma_{k} \sum_{m}\left[n_{k-\sigma}\left(E_{I I I}^{m-\sigma}-E_{I I j}^{\sigma \sigma}\right) \cdot n_{d j}^{\sigma \sigma}-\right. \\
& \left.-\left(1-n_{k-\sigma}\left(E_{I I I}^{m-\sigma}-E_{I I j}^{\sigma \sigma}\right)\right) \cdot n_{\psi m-\sigma}\right]\left|Z_{m j}^{\sigma-\sigma-\sigma 1}\right|^{2}+ \\
& +(k, 1 \leftrightarrow p, 2) \\
& 0=\frac{\partial n_{d j}^{\sigma-\sigma}}{\partial t}=-\Gamma_{k} \sum_{i \sigma}\left[\left(1-n_{k-\sigma}\left(E_{I I j}^{\sigma-\sigma}-\varepsilon_{i}\right)\right) \cdot n_{d j}^{\sigma-\sigma}-\right. \\
& \left.-n_{k-\sigma}\left(E_{I I j}^{\sigma-\sigma}-\varepsilon_{i}\right) \cdot n_{f i \sigma}\right]\left|Y_{j i}^{\sigma-\sigma 1}\right|^{2}- \\
& -\Gamma_{k} \sum_{m \sigma}\left[n_{k \sigma}\left(E_{I I I}^{m \sigma}-E_{I I j}^{\sigma-\sigma}\right) \cdot n_{d j}^{\sigma-\sigma}-\right. \\
& \left.-\left(1-n_{k \sigma}\left(E_{I I I}^{m \sigma}-E_{I I j}^{\sigma-\sigma}\right)\right) \cdot n_{\psi m-\sigma}\right]\left|Z_{m j}^{\sigma \sigma-\sigma 1}\right|^{2}+ \\
& +(k, 1 \leftrightarrow p, 2) \\
& 0=\frac{\partial n_{f i \sigma}}{\partial t}=\Gamma_{k}\left[n_{k \sigma}\left(\varepsilon_{i}\right) \cdot n_{b}-\left(1-n_{k \sigma}\left(\varepsilon_{i}\right)\right) \cdot n_{f i \sigma}\right]\left|X_{i}^{\sigma 1}\right|^{2}+ \\
& +\Gamma_{k} \sum_{j \sigma}\left[\left(1-n_{k-\sigma}\left(E_{I I j}^{\sigma-\sigma}-\varepsilon_{i}\right)\right) \cdot n_{d j}^{\sigma-\sigma}-\right. \\
& \left.-n_{k-\sigma}\left(E_{I I j}^{\sigma-\sigma}-\varepsilon_{i}\right) \cdot n_{f i \sigma}\right]\left|Y_{j i}^{\sigma-\sigma 1}\right|^{2}+ \\
& +\Gamma_{k} \sum_{j}\left[\left(1-n_{k \sigma}\left(E_{I I j}^{\sigma \sigma}-\varepsilon_{i}\right)\right) \cdot n_{d j}^{\sigma \sigma}-\right. \\
& \left.-n_{k \sigma}\left(E_{I I j}^{\sigma \sigma}-\varepsilon_{i}\right) \cdot n_{f i \sigma}\right]\left|Y_{j i}^{\sigma \sigma 1}\right|^{2}+(k, 1 \leftrightarrow p, 2)
\end{aligned}
$$

In these equations we neglect the non-diagonal averages of pseudo-particle operators such as $\left\langle f_{\sigma}^{+} b f_{-\sigma}^{+} d\right\rangle$ etc.. These terms are of the next order in small parameter $\Gamma_{k(p)} / \Delta E$ where $\Delta E$ is the energy difference between any energy states in the coupled QDs. We consider the paramagnetic situation, when conditions $n_{f i \sigma}=n_{f i-\sigma}$, $n_{\psi m \sigma}=n_{\psi m-\sigma}, n_{k \sigma}=n_{k-\sigma}$ and $n_{d j}^{-\sigma-\sigma}=n_{d j}^{\sigma \sigma}$ are fulfilled. System of equations (??) in the stationary case is the linear system, which allows to determine pseudo particle filling numbers, electron filling numbers $n_{e l}(e V)$ and tunneling current $I_{k \sigma}$. 


\section{RESULTS AND DISCUSSION}

The behavior of the total electron occupation of the coupled QDs $n_{e l}(e V)$ and $I-V$ characteristics are presented on the Fig.1 and Fig.2. We first analyze the behavior of the the total electron occupation of the QDs $n_{e l}(e V)$ and $I-V$ characteristics of the considered system for different single electron levels positions relative to the sample Fermi level and various tunneling rates to the contact leads. The bias voltage in our calculations is applied to the sample. Consequently, if both single electron levels are above(below) the Fermi level, all the specific features of the total electron occupation and tunneling current characteristics can be observed at negative(positive) values of $\mathrm{eV}$. In the case when both single electron energy levels are situated above the sample Fermi level [Fig 1] (black line)] we observe the step-like behavior of the total electron occupation. The width and height of the steps are determined by the relation between the system parameters $T, \varepsilon$ and $U$ and $\Gamma=\Gamma_{k}+\Gamma_{p}$. The tunneling current is depicted in [Fig 1 (red line)] as a function of the applied bias. It is clearly evident that even for symmetric tunneling contact the presence of Coulomb interaction leads to the appearance of negative tunneling conductivity. Negative tunneling conductivity was found in the double QDs system connected to the magnetic leads only for particular polarization direction [14], 15]. We also want to point out that negative tunneling conductivity in coupled QDs can observed experimentally [19].

The most interesting result were obtained in the case when both single electron energy levels in the QDs are situated below the Fermi level (Fig 10 (black line), Fig 2ac). In the particular range of the applied bias both for the symmetric $\Gamma_{k}=\Gamma_{p}$ and asymmetric $\Gamma_{k}>\Gamma_{p}$ tunneling contact the total electron occupation demonstrate significant jumps. In a QD without Coulomb interaction the total system occupation can only increase as applied bias increases and passes single electron levels. Quite different situation occurs in a system with strong Coulomb correlations. For zero bias electron filling numbers are determined by equilibrium occupation. But when the increasing bias reaches the energies of multi-electron excited states, the total occupation begins to decrease. Using single-electron language we can say that additional tunneling electrons "push out" electrons from the states below the Fermi level due to Coulomb repulsion. Or one can look at this effect as increasing of probability for electrons to leave the QD due to appearance of several nonelastic channels of tunneling (accompanied with changing of multi-electron states of the QD).

The tunneling current as a function of the applied bias for this case is depicted in Figures 10 (red line) $2 \mathrm{~d}-\mathrm{f}$ and reveals not only the monotonic step-like behavior, but also demonstrates the appearance of negative tunneling conductivity.

Our model also describes the situation, when two QDs (or two levels in one QD) are coupled due to interaction with external field in rotating wave approximation
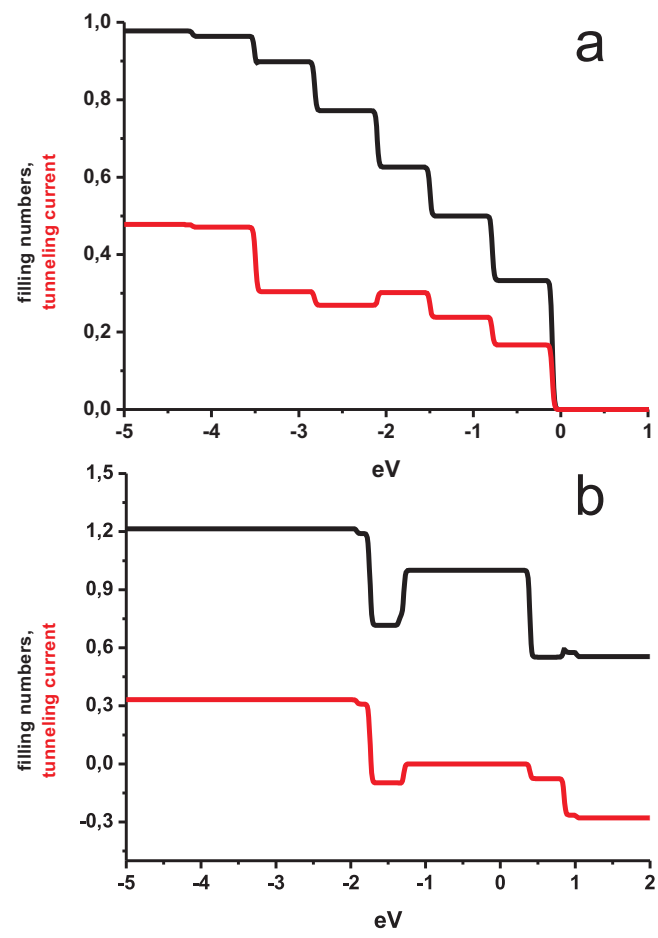

FIG. 1: Fig.1 (Color online) Coupled QDs filling numbers (black line) and tunneling current (red line) as a functions of applied bias voltage in the case of symmetrical tunneling contact $\Gamma_{k}=\Gamma_{p}=0.01$. a). $\varepsilon_{1}=\varepsilon_{2}=0.8, T=0.7, U_{1}=$ $U_{2}=2.0$; b). $\varepsilon_{1}=-0.5, \varepsilon_{2}=-0.6, T=0.3, U_{1}=U_{2}=2.0$

and direct tunneling coupling is negligible. In this case Hamiltonian, which describes interaction with external field, has the form:

$$
\hat{H}_{i n t}==\sum_{\sigma, \beta}\left(d_{\beta}\right)_{12} \epsilon_{\beta} c_{1 \sigma}^{+} c_{2 \sigma}+\text { h.c. }
$$

where $\left(d_{\beta}\right)_{12}$ - are matrix elements for dipole transitions, $\epsilon_{\beta}$ - external field components and $\beta=x, y, z$. So, all the calculations and results remain valid if tunneling transfer amplitude $T$ is replaced by $\sum_{\beta}\left(d_{\beta}\right)_{12} \epsilon_{\beta}=\frac{\Omega}{2}$, where $\Omega$ is a Rabi frequency. Consequently the dependence of tunneling current on the external field intensity at the fixed value of applied bias can be obtained from $I(\Omega)($ Fig 3 ). In the presence of Coulomb interaction the dependence of tunneling current on external field intensity reveals fast switching on and off with the external field intensity increasing. Between the switchings tunneling current amplitude remains nearly constant (see black line in Fig (3). In the absence of Coulomb interaction the tunneling current amplitude smoothly increases with the external field intensity growth. Tunneling current is rapidly switched off at the critical intensity value, which exceeds the critical switching off value for nonzero Coulomb interaction. So, the proposed system can be applied as an effective switch key. 


\section{CONCLUSION}

We investigated tunneling through the system of two strongly coupled QDs weakly interacting with the reservoirs in the presence of Coulomb correlations between localized electrons. In the considered system if electrons number changes due to the tunneling processes, the modification of the energy spectrum is not reduced to the simple adding of Coulomb interaction $U$ per electron. One, two, three or four electrons can be localized in the coupled QDs, each state with fixed total charge and spin projection has it's own energy. Transitions between these states were analyzed in terms of pseudo-particle operators with constraint on the possible physical states of the system. Filling numbers of different multi-electron states, total electron occupation of QDs and $I-V$ characteristics were investigated for different single electron levels positions relative to the sample Fermi level and various tunneling transfer rates.

It was shown that total electron occupation can significantly decrease with increasing of applied bias when both single electron energy levels are situated below the Fermi level contrary to the situation with no correlations. Tunneling electron changes multi-particle states of QDs and pushes out electrons from the state below the Fermi level. We revealed that for some parameter range, the system demonstrates negative tunneling conductivity in certain ranges of the applied bias voltage due to the Coulomb correlations.

We demonstrated, that correlated QDs can be used as an effective current switch key by changing the intensity of the external field.

This work was partly supported by the RFBR and Leading Scientific School grants. The support from the Ministry of Science and Education is also acknowledged.
[1] A.N. Vamivakas, C.-Y. Lu, C. Matthiesen et.al., Nature Letters, 467, (2010), 297.

[2] E.A. Stinaff, M. Scheibner, A.S. Bracker et.al., Science, 311, (2006), 636.

[3] G. Munoz-Matutano, M. Royo, J.I. Climente et.al., Phys. Rev. B, 84, (2011), 041308(R).

[4] K. Kikoin, Y. Avishai, Phys. Rev. B, 65, (2002), 115329.

[5] Y. Goldin, Y. Avishai, Phys. Rev. B, 61, (2000), 16750.

[6] P.I. Arseyev, N.S. Maslova, V.N. Mantsevich JETP Letters, 94(5), (2011), 390.

[7] P.I. Arseyev, N.S. Maslova, V.N. Mantsevich JETP, 115(1), (2012), 141.

[8] P.I. Arseyev, N.S. Maslova, V.N. Mantsevich European Physical Journal B, 85(12), (2012), 410.

[9] J. Paaske, A. Rosch, P. Wolfle, Phys. Rev. B, 69, (2004), 155330.

[10] A. Kaminski, Yu. Nazarov, L. Glazman, Phys. Rev. B,
62, (2000), 8154.

[11] P.A. Orellana, G.A. Lara, E.V. Anda, Phys. Rev. B, 65, (2002), 155317.

[12] R. Lopez, R. Aguado, G. Platero, Phys. Rev. B, 69, (2004), 235305.

[13] V.N. Golovach, D. Loss, Phys. Rev. B, 69, (2004), 245327.

[14] R. Hornberger, S. Koller, G. Begemann et.al., Phys. Rev. B, 77, (2008), 245313.

[15] J. Fransson, Nanotechnology, 17, (2006), 5344.

[16] P. Coleman, Phys. Rev. B, 29, (1984), 3035.

[17] P. Coleman, Phys. Rev. B, 35, (1987), 5072.

[18] N.S. Wingreen, Y. Meir, Phys. Rev. Lett., 49, (1994), 040.

[19] H.W. Liu, T. Fujisawa, T. Hayashi et.al., Phys. Rev. B, 72, (2005), 161305(R). 

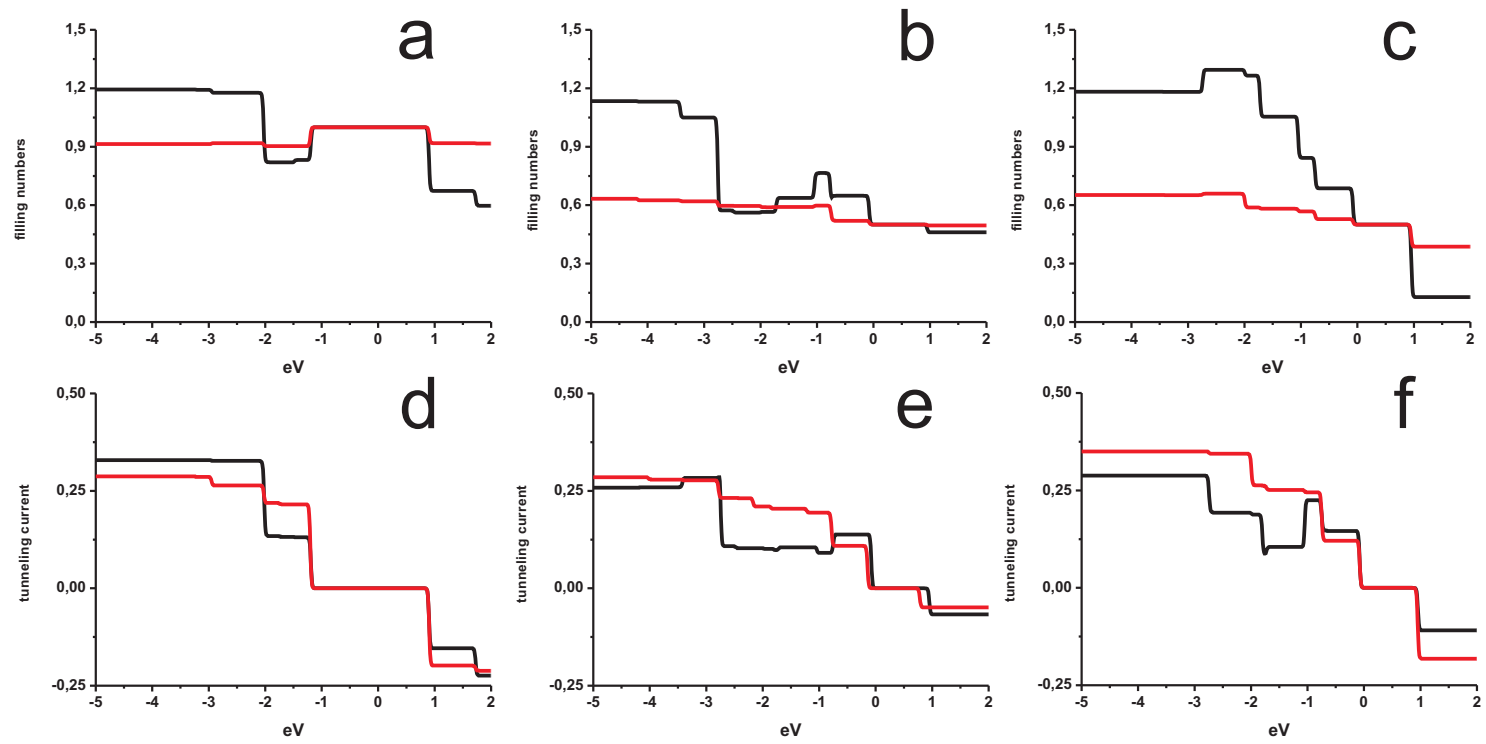

FIG. 2: Fig.2 (Color online) Coupled QDs filling numbers a).-c). and tunneling current d).-f). as a functions of applied bias voltage in the case of symmetrical $\Gamma_{k}=\Gamma_{p}=0.01$ (black line) and asymmetrical $\Gamma_{k}=0.01, \Gamma_{p}=0.1$ (red line) tunneling contact. Parameters $U_{1}=2.0$ and $U_{2}=2.0$ are the same for all the figures. a),d). $\varepsilon_{1}=-0.5, \varepsilon_{2}=-1.2, T=0.8$; b),e). $\varepsilon_{1}=-0.5$, $\left.\left.\varepsilon_{2}=-0.7, T=0.6 ; \mathrm{c}\right), \mathrm{f}\right) . \varepsilon_{1}=-0.7, \varepsilon_{2}=-0.5, T=0.6$.

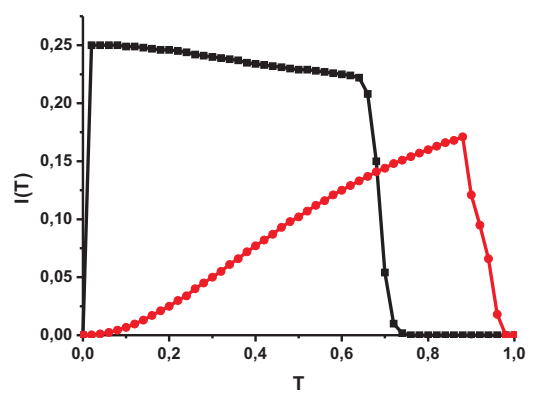

FIG. 3: Fig.3 (Color online) Tunneling current as a functions of Rabi frequency in the case of symmetrical tunneling contact in the presence (black line) and in the absence (red line) of Coulomb interaction. $\varepsilon_{1}=-0.7, \varepsilon_{2}=0.5, e V=-1.0 U_{1}=$ $U_{2}=2.0, \Gamma_{k}=\Gamma_{p}=0.01$ 